\title{
rf conditioning and breakdown analysis of a traveling wave linac with collinear load cells
}

\author{
Qushan Chen, Tongning Hu, Bin Qin, Yongqian Xiong, and Kuanjun Fan \\ State Key Laboratory of Advanced Electromagnetic Engineering and Technology, \\ School of Electrical and Electronic Engineering, Huazhong University of Science and Technology, \\ Wuhan 430074, China \\ Yuanji Pei \\ National Synchrotron Radiation Laboratory, University of Science and Technology of China, \\ Hefei 230026, China
}

(Received 17 December 2017; published 30 April 2018)

\begin{abstract}
Huazhong University of Science and Technology (HUST) has built a compact linac-based terahertz free electron laser (THz-FEL) prototype. In order to achieve compact structure, the linac uses collinear load cells instead of conventional output coupler to absorb remanent power at the end of linac. The new designed structure is confronted with rf breakdown problem after a long time conditioning process, so we tried to figure out the breakdown site in the linac. Without transmitted signal, we propose two methods to analyze the breakdown site mainly based on the forward and the reflected power signals. One method focuses on the time relationship of the two signals while the other focuses on the amplitude. Both the two methods indicate the breakdown events happened at the end of the linac and more likely in the first or the second load cell.
\end{abstract}

DOI: 10.1103/PhysRevAccelBeams.21.042003

\section{INTRODUCTION}

Radio-frequency (rf) breakdown is a common issue in both room-temperature and superconducting accelerating structures and has been extensively studied in many previous works [1-5]. It is critically important to avoid serious rf breakdown because it may cause irreversible damages to accelerating structures and microwave devices. Although the physical mechanism involved in if breakdown phenomenon is not completely understood yet, many theoretical models have been set up to explain the breakdown process [6-9]. There are also considerable simulation works to study the breakdown conditions and track emitted particles in 3D view [10,11]. Experimentally, various methods, such as acoustic sensor [12,13], light detector [14,15], gas analyzer [16], current analysis [17,18], power analysis [19,20], have been developed for $\mathrm{rf}$ breakdown diagnostics.

It is commonly believed that field emission is the main reason for initializing $\mathrm{rf}$ breakdown process [21]. The process can be briefly summarized as follows: field emission is accompanied by gas desorption from the surface of metal structure, and then the neutral gas is ionized by kinetic

\footnotetext{
*bin.qin@mail.hust.edu.cn
}

Published by the American Physical Society under the terms of the Creative Commons Attribution 4.0 International license. Further distribution of this work must maintain attribution to the author(s) and the published article's title, journal citation, and DOI. particles to form a plasma, which forms an electric short to rf power and causing if power reflection. Field emission problem will thus prevent rf structure from operating in high-power mode, as well as obtaining full energy beams. The breakdown rate is strongly correlated with fabrication procedure, processing technique, vacuum condition and $\mathrm{rf}$ power intensity, so extra care needs to be taken for high gradient structure manufacture and process.

Huazhong University of Science and Technology (HUST) has built a compact linac-based terahertz free electron laser (THz-FEL) prototype $[22,23]$. The scientific goal of this project is to explore the use of compact structure to achieve saturated THz lasing. As shown in Fig. 1, the prototype uses a thermionic gun as the electron source, then two standing wave (SW) bunchers modulate the electron beams into short bunches of about $10 \mathrm{ps}$ and accelerate them to 2.6 MeV. The bunches are then injected into a traveling wave (TW) linac and boosted to a maximum energy of $14 \mathrm{MeV}$ within 0.8 -m-long acceleration.

One important feature of the HUST THz-FEL prototype is that the collinear load cells instead of a conventional output coupler are used to dissipate residual rf power at the end of the linac. The choice of collinear load scheme is mainly based on two considerations: one is that it will largely decrease the inner radius of the solenoid wrapping around the linac and make the whole structure compact, as well as the efficiency is enhanced; the other is that comparing to the output coupler scheme, the electromagnetic field inside the collinear load cells is axial symmetry, 


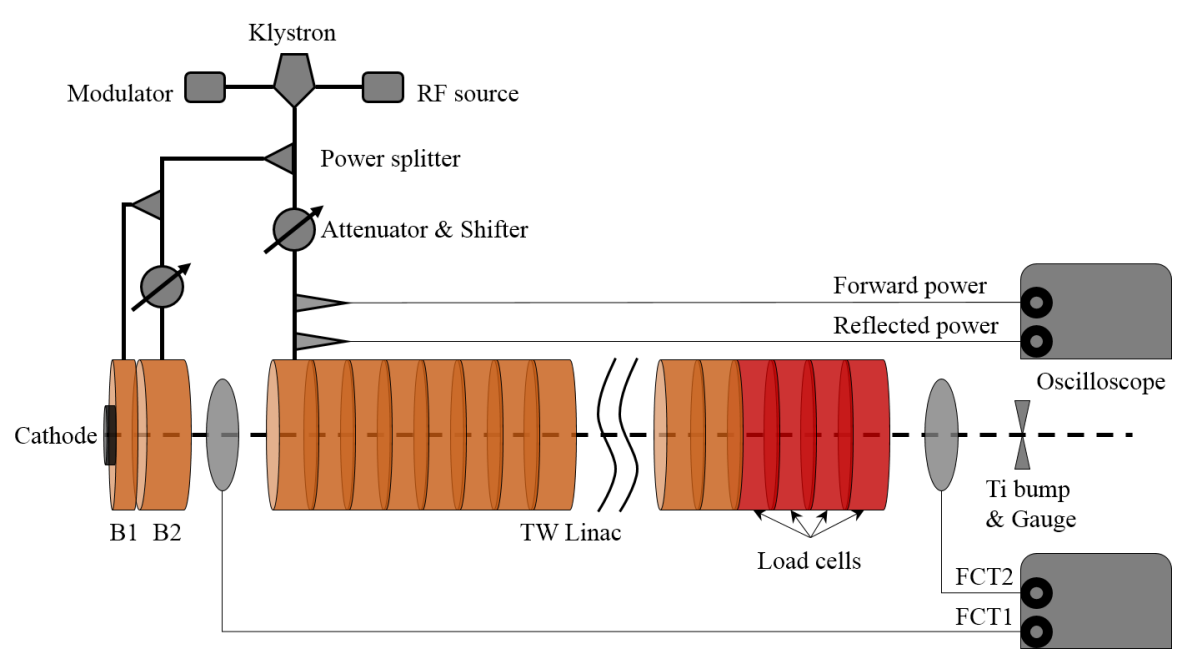

FIG. 1. Schematic view of the rf system and some key rf diagnostic instruments in the HUST compact THz-FEL prototype. B1 and B2 are the two standing wave bunchers.

which is more likely to achieve high quality beams. This project also aims to test the collinear load cells in high power operation and accumulate experience for future applications.

As for safe operation, rf conditioning is a necessary process for the accelerating structure and also an important method to test the collinear load cells. For example, by analyzing the breakdown activities during rf conditioning, it is possible to localize the breakdown point in the linac, which is strongly desirable for future cavity design and processing techniques. However, with the collinear load structure, no transmitted power can be observed during operation, which brings great challenges on the analysis of $\mathrm{rf}$ breakdown events. So the following content will introduce the rf diagnostic instruments of the HUST THz-FEL prototype and focus on how to figure out the rf breakdown points in the linac with limited signals during the conditioning process. It is also expected to get more understanding of rf breakdown phenomena, especially to evaluate the performance of the new designed collinear load linac.

\section{TRAVELING WAVING LINAC WITH COLLINEAR LOAD CELLS}

Generally, a TW linac should have an input and a output coupler for power flow, in which case the inner radius of a solenoid, if there is one installed around the linac, should be large enough to make the coupler pass through. One way to make the solenoid compact and efficient is to remove the output coupler and use collinear load cells to absorb residual rf power at the end of the linac [24]. As a result, the inner wall of the solenoid can wrap the linac tightly.

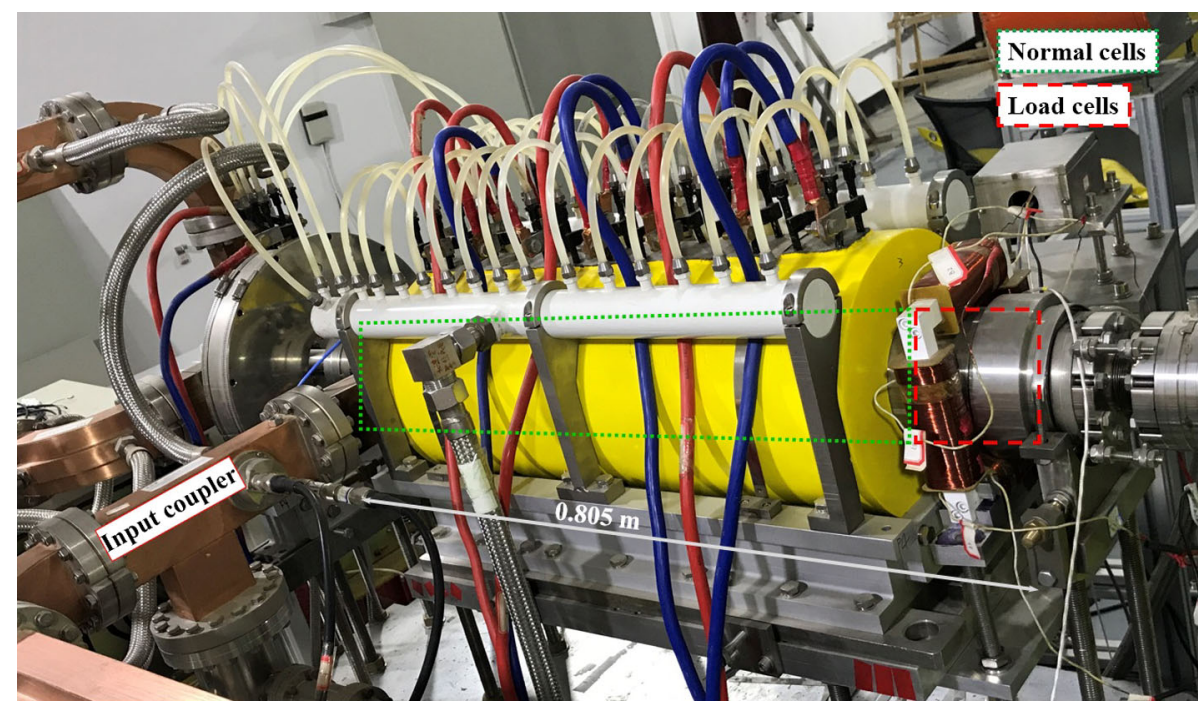

FIG. 2. The compact TW linac in the HUST THz-FEL prototype. The solenoid (painted in yellow) wraps the linac tightly. 


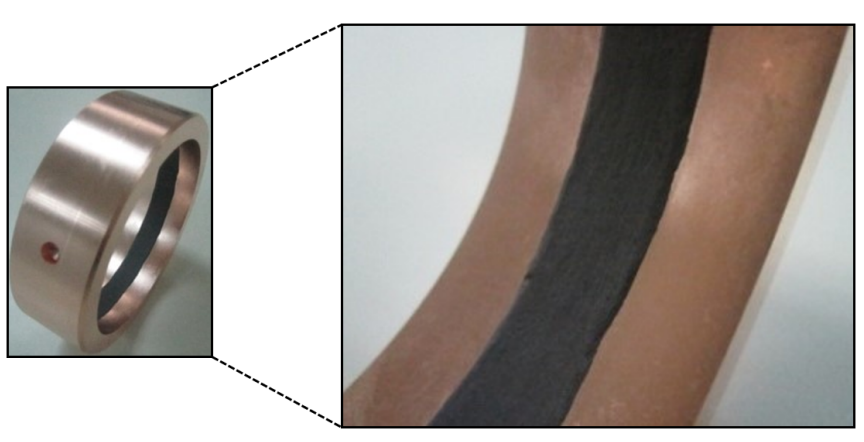

FIG. 3. Load cells with a kind of lossy material (painted in black) coated on the inner wall.

The HUST THz-FEL prototype adopts this compact scheme. As shown in Fig. 2, the total length of the linac, including the upstream and the downstream flanges, is $0.805 \mathrm{~m}$. The linac has no output coupler, so the solenoid wraps the tank tightly. The difference between the outer diameter of the linac and the inner diameter of the solenoid is as low as $1 \mathrm{~mm}$. The TW linac consists of 1 input coupler cell, 19 normal cells (green dot line in Fig. 2) and 4 load cells (red dash line in Fig. 2). The linac is a $2 \pi / 3$-mode type structure and the operation frequency is $2856 \mathrm{MHz}$.

The input coupler cell uses an off-center scheme, which means the beam axis is not exactly coincide with the cell axis and the offset is set to be $1.32 \mathrm{~mm}$ after optimization. This relatively simple and economical scheme can reduce the influence of asymmetry in field strength induced by the side coupler. But it should be noted here that the off-center scheme has little improvement on the symmetry of the phase distribution.

Remanent rf power at the end of the linac is dissipated in the last 4 load cells. The total attenuation strength of the load cells is designed to be $-20 \mathrm{~dB}$ with equal power loss in each of them. The load cells are similar to those normal ones in structure, except that they are coated with some lossy materials on the inner walls (Fig. 3). More detailed report of the lossy material can be found in Ref. [25]. Table I lists some key characteristic parameters of the normal cells and the load cells as a comparison. It can be

TABLE I. Characteristic parameters of the last 4 normal cells and the load cells.

\begin{tabular}{lclr}
\hline \hline Cell No. & $R_{s}(\mathrm{M} \Omega / \mathrm{m})$ & $\alpha(1 / \mathrm{m})$ & \multicolumn{1}{c}{$Q$} \\
\hline 16 & 64.77 & 0.275 & 13916.1 \\
17 & 64.89 & 0.280 & 13915.5 \\
18 & 65.00 & 0.285 & 13914.9 \\
19 & 65.13 & 0.290 & 13914.2 \\
20 & 4.63 & 4.063 & 993.1 \\
21 & 3.30 & 5.699 & 708.0 \\
22 & 1.96 & 9.625 & 419.2 \\
23 & 0.41 & 46.42 & 86.9 \\
\hline \hline
\end{tabular}

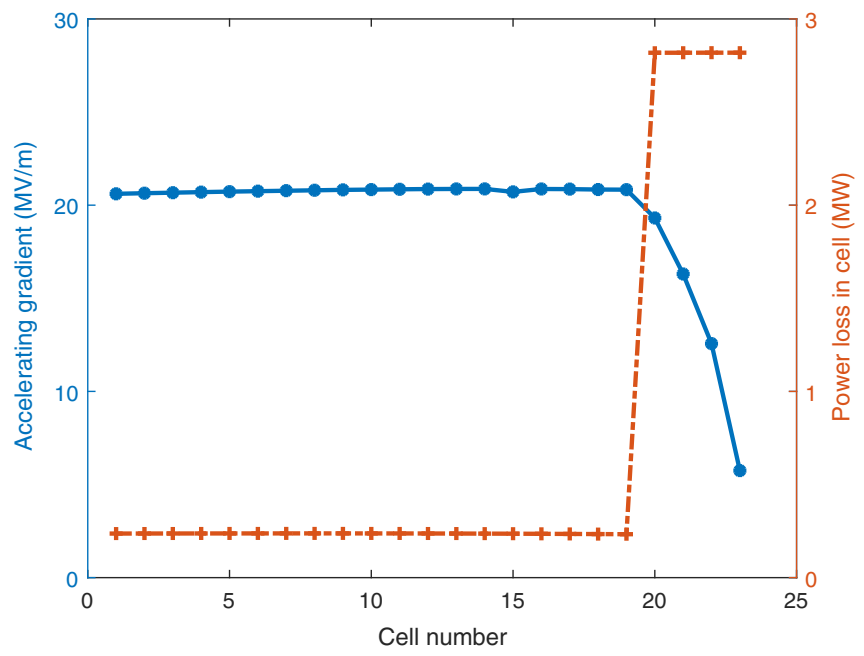

FIG. 4. Accelerating gradient and power loss in each cell of the TW linac when the input power is $16 \mathrm{MW}$.

found that both the shunt impedance $\left(R_{S}\right)$ and the quality factor $(Q)$ of the load cells are much smaller than that of the normal ones, thus the attenuation factors $(\alpha)$ of the load cells are much larger. The attenuation factors of the load cells are controlled by adjusting the volume of the lossy material coated on the inner wall. And finally, the attenuation factors are selected to achieve equal power loss among the load cells and total attenuation of $-20 \mathrm{~dB}$. It is worth noting here that this feature is independent of the strength of input power. Figure 4 shows the accelerating gradient and power loss in each cell of the linac when the input pulse power is $16 \mathrm{MW}$, which is the upper bound for the linac. In this case, the pulse power dissipated on the normal and load cells are 4.6 and 11.3 MW respectively.

Spiral water tube around the linac is designed to achieve good cooling effect. As the load cells have higher power loss, the cooling water flows from the end to the head of the linac. According to the thermodynamic analysis, temperature rise of the normal cells and the load cells can be controlled within $0.5^{\circ} \mathrm{C}$ and $1.0^{\circ} \mathrm{C}$ respectively. Factory rf testing shows the linac has a VSWR (voltage standing wave ratio) of 1.01 at the operating frequency $\left(f_{0}=2856 \mathrm{MHz}\right)$ and a VSWR $<1.2$ within a bandwidth of $1.5 \mathrm{MHz}$.

\section{III. rf CONDITIONING OF THE LINAC}

\section{A. rf system}

The schematic view of the rf system has been shown in Fig. 1. The rf source from Rohde and Schwarz generates the original signal to drive a solid state amplifier. The amplifier (not shown in Fig. 1) is triggered by a master clock system to provide pulse power of about $800 \mathrm{~W}$. Then the amplifier together with the modulator drives the klystron (E3708 from Toshiba) to provide the final $\mathrm{rf}$ power, which is further divided into three parts through two power splitters to drive the two bunchers and the 
linac. The pulse length and repetition rate can be adjusted by the master clock system while the power strength from the klystron is controlled by the driving voltage of the modulator. Actually, in practical operation the rf power strength from the klystron is usually kept fixed and there are two sets of attenuators and phase shifters being used to adjust the strength and phase of input power to the second buncher and the linac (as shown in Fig. 1). The power phase of the second buncher mainly influences the bunching effect while the power strength of the linac mainly influences the final beam energy. As the system requires the beam energy varying from 6 to $14 \mathrm{MeV}$, the input power to the linac should be adjusted accordingly.

\section{B. Diagnostic instruments}

Strong reflected power or low transmitted power is the most straightforward phenomenon in rf breakdown events. In the meantime, breakdown events will usually accompany abnormal emission current and gas desorption. So rf power, current and vacuum are the most common signals to be detected during rf conditioning process.

In the HUST THz-FEL prototype, no transmitted power signal can be detected, so the rf breakdown analysis is mainly based on the forward and the reflected power signals. As shown in Fig. 1, the forward and the reflected power signals are connected to the same oscilloscope. The oscilloscope works in the persistence mode, which means all waveform received by the oscilloscope will be kept on the screen. This setting will help to record all abnormal signals and present a comparison with normal signals. In addition, there are two fast current transformers (FCT1 and FCT2 in Fig. 1) installed upstream and downstream the linac respectively. The two FCTs are connected to another oscilloscope with two separate shielded coaxial cables. The calibration factors of the two FCTs are both $4.3 \mathrm{~V} / \mathrm{A}$. It should be noted that the FCT1 can detect current signals from the bunchers as well as that from the linac. But it will generate signals with opposite signs. Assuming a positive signal corresponds to an electron beam passing through the FCT from upstream to downstream, it has been observed that the FCT1 gave obvious positive signals when a rf breakdown event happened in the upstream bunchers. As the electron trajectories during field emission or multipacting are randomly distributed, some emitted electrons from the linac may go upstream and detected by the FCT1. If this is the case, the FCT1 will give a negative signal, which can be easily distinguished from the buncher signals.

Figure 5 displays a record of a serious discharge event. As all channels are set to reverse phase mode, the "Tor1" channel obviously gives a negative signal, which indicates the detected current was emitted from the downstream linac. One can also find that the emission current detected by the FCT1 and FCT2 reaches 0.45 and 1.39 A respectively, indicating huge emission current. Apart from this, we can also infer that the discharge point related to Fig. 5

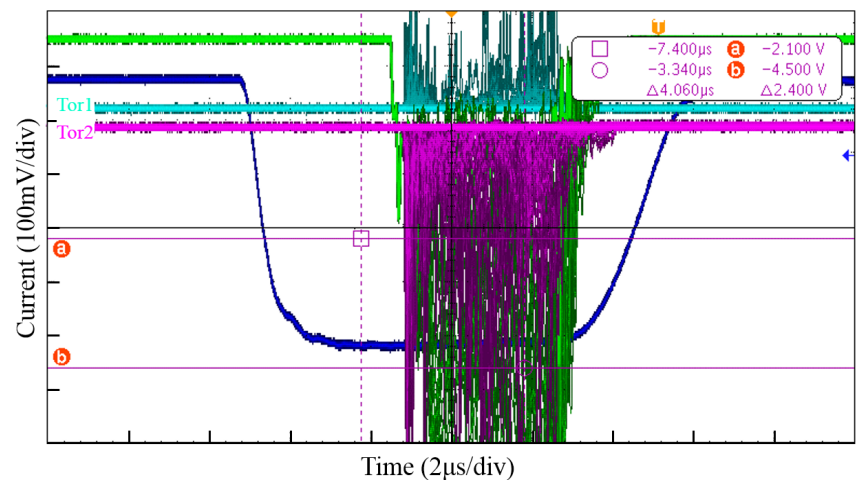

FIG. 5. Record of a serious discharge event. Tor1: readout of the FCT1; Tor2: readout of the FCT2. All channels are set to reverse phase mode. The unit of the ordinate only applies for the Tor1 and Tor2 channels.

locates close to the entrance of the linac, because it is hard for electrons passing through the linac opposite to the direction of TW power. Detailed analysis of this dynamic process needs complicated particle tracking program [26] and is beyond the scope of this paper.

A cold cathode gauge and a titanium pump are installed just downstream the FCT2. Usually, the vacuum signal is slower than the current signal and the power signal, so it is used as a crosscheck to determine whether discharge happens or not. The gauge signal also works as an interlock for the whole system. If the gauge reading is higher than $5 \times 10^{-5} \mathrm{~Pa}$, the power and the current signals will be saved into a local workstation. If the gauge reading is higher than $10^{-4} \mathrm{~Pa}$, the master clock system, as well as the rf power, will be shut down to protect the rf system.

\section{C. rf conditioning process}

The rf conditioning process of the HUST linac is divided into two stages. The first stage is the low-power conditioning, in which case the input power to the linac is just a few megawatts and the axial electric field is less than $10 \mathrm{MV} / \mathrm{m}$. The low-power conditioning is also recognized as multipacting region, for the resonance condition for multipacting is well satisfied [21]. In this stage, vacuum deterioration and intensive discharge current were the two most notable phenomena. Sometimes the discharge current detected by the FCT2 could reach $1 \mathrm{~A}$. In the meantime, negative signal could be found on the FCT1, which indicated some discharged electrons run upstream through the linac and detected by the FCT1 (Fig. 5). Power reflection was another bothersome problem in this stage. As there is no circulator in the rf system, the reflected power to the klystron should be strictly controlled within safe level. Based on this consideration, the rf power to the linac was increased very carefully and this conditioning process lasted for several weeks, until the vacuum kept steady in low level after long-time power 
TABLE II. Comparison of typical and maximum rf parameters of the linac during operation.

\begin{tabular}{lcr}
\hline \hline Parameters & Typical & Maximum \\
\hline rf frequency $(\mathrm{MHz})$ & 2856 & 2856 \\
Pulse power $(\mathrm{MW})$ & 11 & 16 \\
Pulse length $(\mu \mathrm{s})$ & $2-4$ & 4 \\
Repetition rate $(\mathrm{Hz})$ & 1 & 50 \\
\hline \hline
\end{tabular}

feed in. Anyway, one can always go through this stage safely, if only the ramping of rf power is sufficient slow and of course the conditioning process will last very long.

The second stage is the high-power conditioning, in which case the input power to the linac is higher than $5 \mathrm{MW}$ and the axial electric field gradually approaches the working point $(20 \mathrm{MV} / \mathrm{m})$. In this process field emission happened frequently and sometimes it would induce rf breakdown. The recorded abnormal emission current were usually on the level of hundreds of mA. At the same time, strong power reflection and notable vacuum deterioration could be detected. These phenomena clearly indicated if breakdown events. Generally, the rf breakdown rate should gradually decrease with the conditioning goes on, but it seemed very hard for the HUST linac to reach an acceptable breakdown rate. The FEL light source requires a low breakdown rate $(<1 \mathrm{e}-6 /$ pulse $)$ while the conditioning process gave a rate on the level of $1 \mathrm{e}-4 /$ pulse.

As the stability of the modulator and capacity of the water cooling system can not support $50 \mathrm{~Hz}$ operation, the repetition rate is set to $5-20 \mathrm{~Hz}$ during high-power conditioning and 1 or $2 \mathrm{~Hz}$ during beam operation. Table II lists the typical and maximum operational rf parameters of the linac. It can be seen from Table II that the typical power strength and repetition rate are much smaller than the maximum values. It is because under the maximum value operation, the rf breakdown rate will increase and the electron bunches becomes unstable. After several weeks rf conditioning process, the linac was still not very suitable for high power operation because of unacceptable $\mathrm{rf}$ breakdown rate. Then for safe consideration, we went back to high-power conditioning and desired to find the breakdown points in the linac.

\section{ANALYSIS OF rf BREAKDOWN EVENTS}

\section{A. Record of rf breakdown events}

A typical record of discharge current during the highpower conditioning is shown in Fig. 6. Different from the multipacting cases, the discharge current during the highpower conditioning is much lower. It can be calculated from Fig. 6 that the current detected by the FCT2 is on the level of $0.1 \mathrm{~A}$ while there is no obvious signal detected by the FCT1.

Figure 7 displays the record of power signal from the same rf breakdown event. As different channel has different

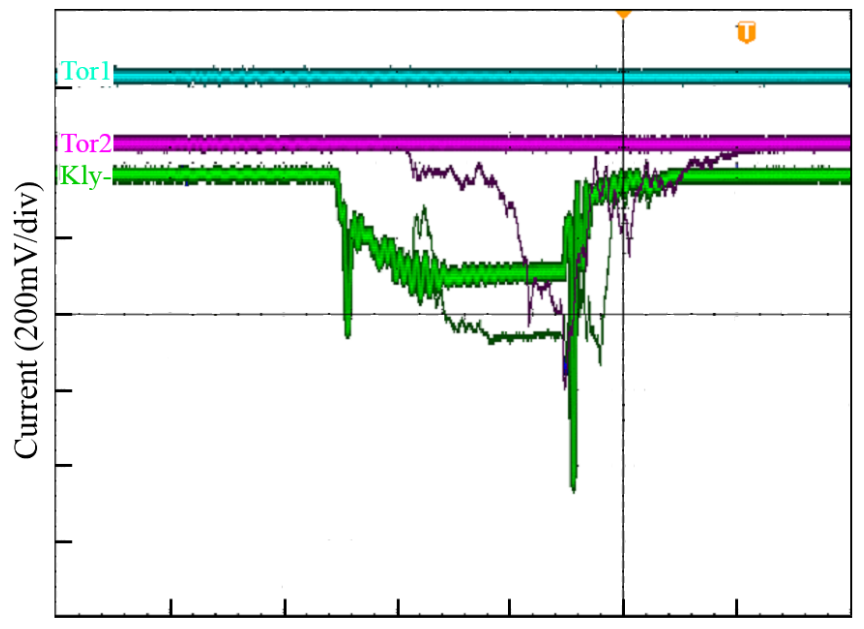

Time $(2 \mu \mathrm{s} / \mathrm{div})$

FIG. 6. Abnormal emission current for one rf breakdown event during high-power conditioning. Tor1: readout of the FCT1; Tor2: readout of the FCT2; Kly-: reflected power to the klystron. The unit of the ordinate only applies for the Tor1 and Tor2 channels.

scaling factor and attenuator, it is no meaningful to identify the unit of the ordinate. But the power strength corresponding to each channel will be revealed in the following subsections. It can be seen clearly the increase of reflected power from the linac. There is also slight increase of forward power of the klystron and the linac. It may come from the standing wave effect in the waveguide after a sudden increase of the reflected power. Most important, it is worth to note the time relationship between the forward and the reflected signals. The reflected signal ("Acc-" in Fig. 7) has a time delay of $1.3 \mu$ s referred to the forward signal ("Acc+" in Fig. 7). Besides, the pulse length of the

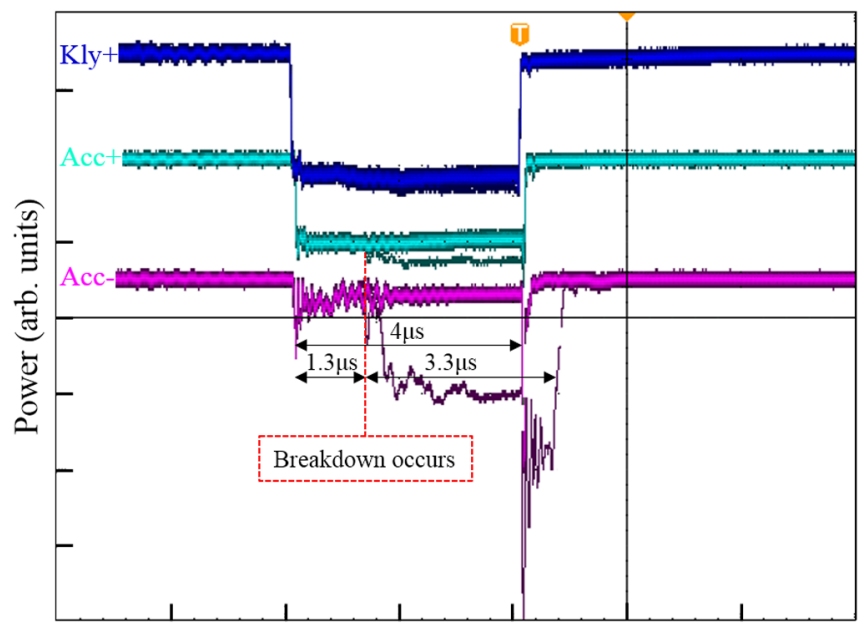

Time $(2 \mu \mathrm{s} / \mathrm{div})$

FIG. 7. rf wave distortion for one rf breakdown event during high-power conditioning. Kly+: forward power from the klystron; Acc +: forward power of the linac; Acc-: reflected power from the linac. 
forward signal is $4 \mu \mathrm{s}$, which is determined by the master clock system, while that of the reflected signal is $3.4 \mu \mathrm{s}$. In the following, detailed analysis will go to the forward and reflected signals and try to find the rf breakdown points in the linac.

\section{B. Time delay based method}

The time delay based method is to use the time relationship, both delay and length, of the forward and reflected signals to compute the rf breakdown point. Figure 8 helps to illustrate the time delay based method.

Consider a rf pulse of length $W_{1}$ propagates downstream in the linac tube. When the pulse arrives at some position, discharge happens and a cluster of electrons are emitted due to high local field. From the pulse's point of view, it is assumed that the discharge point locates at $\left(W_{1}-W_{2}\right)$ referred to the head of the pulse. Then the downstream part of the pulse, which is of length $\left(W_{1}-W_{2}\right)$ propagates normally, while the upstream part of the pulse, which is of length $W_{2}$ is reflected. Once the reflected power signal is observed, there should be a time delay of $D$ referred to the forward one. The time delay is caused by two parts: one is the difference in length between the forward pulse and the reflected pulse; the other is the filling time from the detection point to the discharge point. Based on these analysis, we have

$$
D=\left(W_{1}-W_{2}\right)+2 T_{B P},
$$

where $T_{B P}$ is the filling time from the entrance of the linac to the cell where the discharge event happens.

After measuring the time delay and pulse length of the forward and reflected signals, the filling time $T_{B P}$ can be obtained according to Eq. (1). Then we can calculate the cell number of the discharge cell with

$$
T_{B P}=\sum_{k=1}^{X} L_{0} / v_{g}^{k},
$$

where $L_{0}$ is the length of each cell, $V_{g}^{k}$ is the group velocity of the $k$ th cell, $X$ is the cell number indicating the

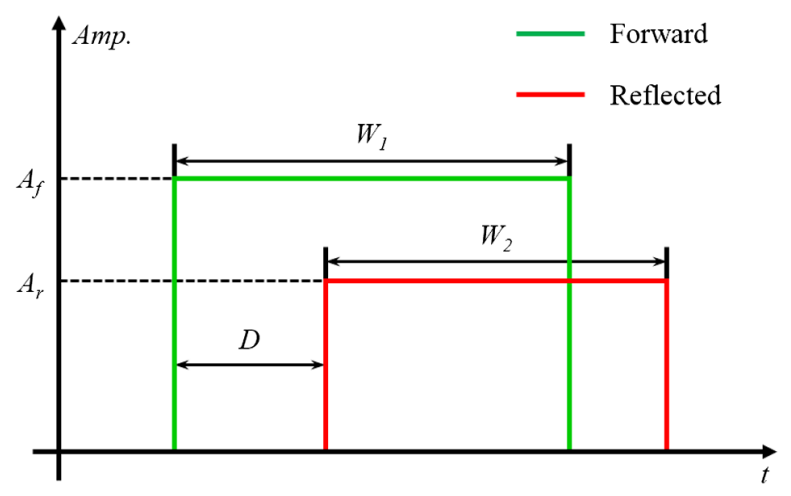

FIG. 8. Illustration of rf power when breakdown occurs. discharge cell. For a constant phase velocity structure, the cell length $L_{0}$ is the same along the structure. As for the case of the HUST linac, it is operated in $2 \pi / 3$ mode and the resonant frequency is $2856 \mathrm{MHz}$, so the cell length is $L_{0}=\lambda / 3=34.99 \mathrm{~mm}$. One can increase the value of $X$ from 1 to 23 step by step until the calculated $T_{B P}$ is just larger than that calculated from Eq. (1).

Now referring to Fig. 7, the pulse width of the forward signal is $W_{1}=4 \mu \mathrm{s}$ while that of the reflected signal (abnormal wave) is $W_{2}=3.3 \mu \mathrm{s}$. The time delay between them is $D=1.3 \mu \mathrm{s}$. According to Eq. (1), the filling time from the entrance to the discharge cell is $T_{B P}=0.3 \mu \mathrm{s}$. Then with Eq. (2), the cell number $X$ would be 22, indicating the discharge event happens in the third load cell.

To be noted, the time delay based method reported here can just give a rough estimation of the discharge location. It is because the uncertainty of the time measurement in Fig. 7 is on the level of $0.1 \mu \mathrm{s}$. With some simple analysis, it gives the uncertainty of $T_{B P}$ to be $0.087 \mu \mathrm{s}$. As the filling time of one cell of the HUST linac is around $0.015 \mu \mathrm{s}$, the uncertainty of $X$ would be 5 or 6 . So, one can only conclude that the discharge happens at the end of the linac while unable to determine the exact cell number. Actually, Fig. 7 is obtained under a sampling rate of 500 million samples per second, which corresponds to a time resolution of $0.002 \mu \mathrm{s}$. But it is a pity that the original data was only saved in figure format. Otherwise the uncertainty of the pulse width measurement can be greatly decreased. $0.1 \mu \mathrm{s}$ is the best uncertainty that one can obtained from Fig. 7. Anyway, the time delay based method still shows its ability to determine the exact discharge location in a collinear load structure with only forward and reflected power signals.

\section{Amplitude comparison based method}

Amplitude comparison based method is somewhat straightforward to understand. Still referring to Fig. 8, the amplitude of the forward power at the entrance of the linac is $A_{f}$. Please note here that the amplitude is read by a rf detector and proportional to the power strength rather than the electric strength. When the forward power induces a rf breakdown event at some point, the power is divided into three parts:

$$
\left\{\begin{array}{l}
P_{t}=m_{1} P_{f} e^{-2 L_{0}\left(\sum_{k=1}^{N} \alpha_{k}\right)} \\
P_{l}=m_{2} P_{f} e^{2 L_{0}\left(\sum_{k=1}^{X} \alpha_{k}\right)} \\
P_{r}=\left(1-m_{1}-m_{2}\right) P_{f} e^{-4 L_{0}\left(\sum_{k=1}^{X} \alpha_{k}\right)}
\end{array},\right.
$$

where $P_{t}$ is the portion that still transmits forward and detected at the end of the linac, if there is a output coupler and a detector installed there; $P_{l}$ is the portion dissipated by emitted electrons; $P_{r}$ is the rest part that is reflected and detected at the entrance of the linac; $P_{f}$ is the forward power strength at the entrance; $\alpha_{k}$ is the attenuation factor 
of the $k^{\text {th }}$ cell; $N$ is the total number of cells of the linac; $m_{1}$ and $m_{2}$ are the two factors indicating the power portion of the transmitted part and the lossy part at the discharge point.

As for the HUST collinear load linac, only $P_{f}$ and $P_{r}$ are measurable. From Eq. (3), the ratio of the forward and reflected signals has a direct relationship with the discharge location $X$.

$$
\frac{A_{r}}{A_{f}}=\left(1-m_{1}-m_{2}\right) e^{-4 L_{0}\left(\sum_{k=1}^{X} \alpha_{k}\right)} .
$$

Theoretically, after measuring the amplitude of the forward and the reflected signals, as illustrated in Fig. 8 $\left(A_{f}\right.$ and $A_{r}$ ), one can calculate the discharge location $X$ according to Eq. (4). But still there are two problems related to this idea. The first one is the determination of $m_{1}$ and $m_{2}$. It is known from the dynamic process of rf breakdown that power reflection is caused by ionization cloud at the discharge location. So qualitatively, more emitted electrons will result in stronger power reflection, as well as lower power transmission. From this point of view, $m_{1}$ and $m_{2}$ seem to counterbalance with each other. There is also report that nearly $100 \%$ of $\mathrm{rf}$ power fed into the linac is reflected during rf breakdown [2], indicating $m_{1}$ and $m_{2}$ together still occupy a small portion of the total power. However, to be rigorous, Eq. (4) can be transformed into an inequation, from which the upper bound of $X$ can be obtained.

$$
\sum_{k=1}^{X} \alpha_{k} \leq \frac{1}{4 L_{0}} \ln \frac{A_{f}}{A_{r}} .
$$

Now the other problem is the exact measurement of $A_{r}$. Usually the power flow during rf breakdown is very tricky and the observed reflected signal wave is seriously distorted. It is impossible to obtain the ideal rectangular pulse as plotted in Fig. 8. Nevertheless, from the above analysis, smaller $m_{1}$ and $m_{2}$ will result in more reliable $X$. So it is reasonable to select the peak point of the reflected signal as $A_{r}$, regardless of the front edge and the trailing edge.

Still referring to Fig. 7, a relatively clear rectangular pulse is observed in the "Acc-" channel. The measured forward power and reflected power (in rf breakdown case) of the linac are 6.55 MW and 1.03 MW respectively. Then according to Eq. (5), the one-way attenuation strength from the entrance to the discharge location should be at most 13.22. Figure 9 demonstrates how the cumulative attenuation strength grows along the linac. It can be easily found that $X<21$, which indicates the discharge point locates before the 21 st cell.

The amplitude comparison based method can just give the upper bound rather than a confidence interval of the result. However, as mentioned above, the transmitted and lossy power usually occupy a small part of the total power from empirical results. So the calculated upper bound is almost close to the real location.

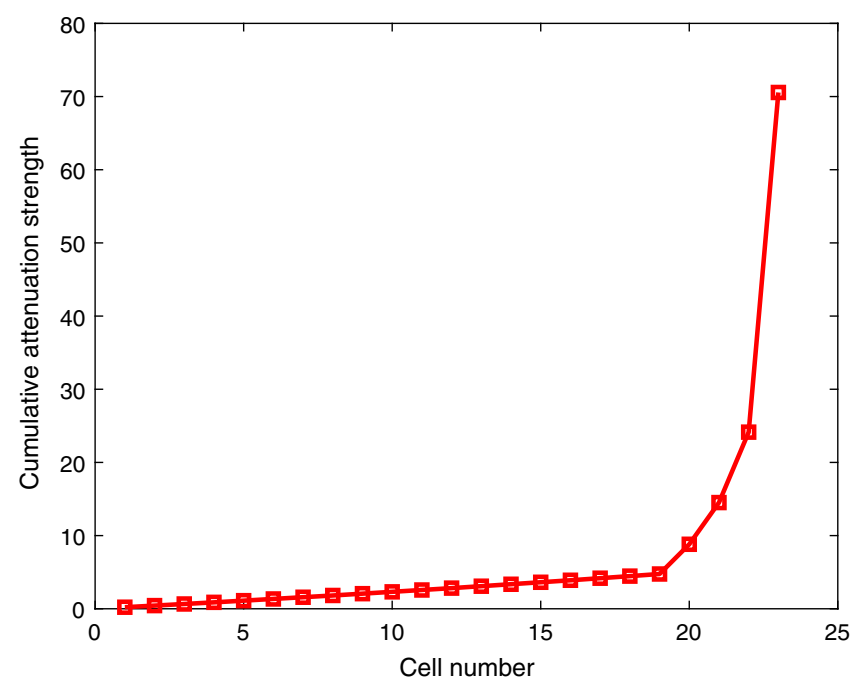

FIG. 9. Cumulative attenuation strength along the linac, which is defined as $\sum_{k=1}^{X} \alpha_{k}$.

\section{CONCLUSION AND DISCUSSION}

Both the two methods reported in this paper indicate the recorded rf breakdown events happened at the end of the linac, although due to the limited data and time resolution we can not tell the exact cell in which it happened. Actually, the oscillators can generate data files of much better time resolution. So, we are planning to set up an auto-record program to instantly communicate with the oscillators. Data of all channels will be saved to a local disk once there is an abnormal signal detected. In this case, the time resolution will be mainly determined by the sample rate of the oscillators $(5 \mathrm{GSa} / \mathrm{s}$ corresponds to a time resolution of $2 \mathrm{~ns}$ in our device).

Besides, one can find from Fig. 2 that the solenoid wrapping around the linac consists of three independent coils. We have tried to change the current of each coil in turn during the $\mathrm{rf}$ conditioning process and found that the current variation of the last coil would obviously influence the breakdown rate and the emission current. As the kinetic energy of the initial emitted electrons from the copper wall is relatively low, their trajectories would be greatly influenced by the magnetic flux of the coils. From this point of view, the coil current might influence the breakdown behavior if the breakdown site locates close to the coil. So this phenomenon also in some degree indicates the breakdown point located at the end of the linac.

In order to achieve compact structure, the HUST THzFEL prototype uses collinear load cells instead of conventional output coupler to absorb remanent power at the end of the linac. The load cell is different from the normal ones by coating a kind of lossy material on the inner walls. This scheme is a new attempt to our group and its high power $\mathrm{rf}$ performance has not been tested before. Combining all the above experimental results and analysis, it is more likely to conclude that the collinear load cells should be responsible 
for the rf breakdown events. Based on this point, some improvements of processing technique may be needed for the collinear load cells in future applications. As it can be found from Fig. 3 the defective uniformity of the lossy material on the copper wall, one important aspect is to enhance the uniformity of the coating process, although it is a great challenge for our engineers.

\section{ACKNOWLEDGMENTS}

This work is supported by the National Science Foundation of China under the Contracts of No. 11705062 and No. 11375068, as well as the China Postdoctoral Science Foundation under the Contract of No. 2017M622440. The authors would like to thank W. Chen, J. Li et al., for their continuous efforts on machine operation and data acquisition. The authors would also like to give full respect to the engineers from National Synchrotron Radiation Laboratory (NSRL) for their great help in the experimental setup.

[1] H. Matsumoto et al., RF breakdown studies on an S-band disk loaded structure, Proceedings of PAC1987, Washington, USA (1987), pp. 1654-1656.

[2] A. Palaia et al., Effects of rf breakdown on the beam in the Compact Linear Collider prototype accelerator structure, arXiv:1301.4673v2.

[3] T. Garvey et al., The rf power coupler development programme at LAL-Orsay and DESY-Hamburg for TESLA and the European X-FEL, International Congress on Optics and Opto-Eletronics, Warsaw, Poland (2005).

[4] F. Wang, C. Adolphsen, and C. Nantista, Study of radio frequency breakdown in pressurized L-band waveguide for the International Linear Collider, Appl. Phys. Lett. 103, 104106 (2013).

[5] V. Dolgashev, S. Tantawi, Y. Higashi, and B. Spataro, Geometric dependence of radio-frequency breakdown in normal conducting accelerating structures, Appl. Phys. Lett. 97, 171501 (2010).

[6] P. B. Wilson, A theory of the rf surface field for various metals at the destructive breakdown limit, Report No. SLAC-PUB-12354, 2007.

[7] A. Grudiev, S. Calatroni, and W. Wuensch, New local field quantity describing the high gradient limit of accelerating structures, Phys. Rev. ST Accel. Beams 12, 102001 (2009).

[8] A. Hassanein et al., Effects of surface damage on rf cavity operation, Phys. Rev. ST Accel. Beams 9, 062001 (2006).

[9] Z. Insepov, J. Norem, and S. Veitzer, Atomistic selfsputtering mechanisms of rf breakdown in high-gradient linacs, Nucl. Instrum. Methods Phys. Res., Sect. B 268, 642 (2010).

[10] M. A. Gusarova et al., Multipacting simulation in input power couplers of electron linear accelerator, Problems of Atomic Science and Technology 6, 96 (2013).
[11] R. Gennaday et al., Simulation of multipacting in SC low beta cavities at FNAL, Proceedings of IPAC15, Newport News, USA (2015), pp. 579-581.

[12] F. Le Pimpec, J. Frisch, K. Jobe, D. McCormick, J. Nelson, M. Ross, and T. Smith, An acoustic sensor system for localizing RF breakdown in warm copper accelerating structures, Nucl. Instrum. Methods Phys. Res., Sect. A 582, 345 (2007).

[13] M. Gangeluk et al., Acoustic monitoring system of RFbreakdown inside the electrodynamic structures at Kurchatov SR source accelerators, Proceedings of the Fourth European Particle Accelerator Conference EPAC 94, London, England (World Scientific, River Edge, NJ, 1994), p. 1986.

[14] J. W. Kovermann et al., Advanced experimental techniques for RF and DC breakdown research, Proceedings of the 11th European Particle Accelerator Conference, Genoa, 2008 (EPS-AG, Genoa, Italy, 2008), p. 775.

[15] H. Tomizawa, T. Taniuchi, H. Hanaki, Y. Igarashi, S. Yamaguchi, and A. Enomoto, Spectrographic approach for the diagnosis of rf breakdown in accelerating rf structures, Appl. Surf. Sci. 235, 214 (2004).

[16] F. Wang, Multipacting electron signal behavior, Nucl. Instrum. Methods Phys. Res., Sect. A 698, 147 (2013).

[17] M. Jacewicz, V. Ziemann, T. Ekelöf, A. Dubrovskiy, and R. Ruber, Spectrometers for RF breakdown studies for CLIC, Nucl. Instrum. Methods Phys. Res., Sect. A 828, 63 (2016).

[18] M. Johnson, R. Ruber, V. Ziemann, and H. Braun, Arrival time measurements of ions accompanying RF breakdown, Nucl. Instrum. Methods Phys. Res., Sect. A 595, 568 (2008).

[19] G. A. Loew and J. W. Wang, RF breakdown studies in room temperature electron linac structures, Report No. SLAC-PUB-4647 (1988).

[20] J. W. Wang and G. A. Loew, Field emission and RF breakdown in high-gradient room-temperature linac structures, Report No. SLAC-PUB-7684 (1997).

[21] T. P. Wangler, RF Linear Accelerators, 2nd completely revised and enlarged edition (WILEY-VCH, New York, 2008), ISBN: 978-3-527-40680-7.

[22] B. Qin, P. Tan, L. Yang, and X.L. Liu, Design considerations of a planar undulator applied in a terahertz FEL oscillator, Nucl. Instrum. Methods Phys. Res., Sect. A 727, 90 (2013).

[23] Q. Chen, B. Qin, P. Tan, T. Hu, Y. Pei, and F. Zhang, Design of RF chopper system for improving beam quality in FEL injector with thermionic gun, Nucl. Instrum. Methods Phys. Res., Sect. A 755, 78 (2014).

[24] K. Jin, Y. J. Pei, D. M. Jiang, and Y. Z. Liu, Collinear load study for X-band linear accelerator structure, Nucl. Instrum. Methods Phys. Res., Sect. A 488, 473 (2002).

[25] Y. Sun, L. Shen, X. Wang, Z. Shu, and Y. Pei, Numerical analysis and design of collinear load for S-band linac based on FeSiAl material, Nucl. Instrum. Methods Phys. Res., Sect. A 623, 883 (2010).

[26] V. A. Dolgashev and S. G. Tantawi, Simulations of currents in X-band accelerator structures using 2D and 3D particlein-cell code, Proceedings of the Particle Accelerator Conference, Chicago, IL, 2001 (IEEE, New York, 2001), p. 3807. 